\title{
SUFICIENCIA DE LA DIETA Y COMPOSICIÓN CORPORAL EN UN GRUPO DE NIÑOS DE 11-14 AÑOS DE DOS CLUBES DEPORTIVOS EN SANTIAGO DE CHILE
}

\author{
ADEQUACY OF THE DIET AND BODY COMPOSITION \\ IN A GROUP OF 11-14 YEARS OLD CHILDREN FROM TWO \\ SPORT CLUBS IN SANTIAGO DE CHILE
}

\author{
Yéssica Liberona Z., Oscar Castillo V., Jaime Rozowski N. \\ Unidad de Intervención Nutricional, Departamento de Nutrición Diabetes y Metabolismo, \\ Facultad de Medicina, Pontificia Universidad Católica de Chile, Santiago, Chile.
}

\begin{abstract}
The information available on food consumption in different population groups is very limited in Chile. Objective: To assess the diet of schoolchildren who on a regular basis perform physical activity. Method: We evaluated 105 schoolchildren aged 11-14 years who regularly attend two sport clubs and who, on the average, perform 8 hours of programmed activity per week. Anthropometric evaluation included height, weight, tricipital and subscapular skinfold thickness and diet was evaluated by means of a 24-hour dietary recall. Socioeconomic level was determined by the ESOMAR method. Results: As expected, height and weight increased progressively with age. Percentage of body fat increased between 11 and 13 years old and significantly decreased by age 14 years. Nutritional status was normal in $86 \%$ of the children and only $14 \%$ were overweight. There were no obese children in the group. Macronutrient and energy intake were adequate for the age except for the low consumption of potassium and calcium. Conclusion: Children who perform exercise regularly show a low prevalence of overweight and no obesity although their diet was similar to other schoolchildren who show an elevated prevalence of overweight and obesity, most probably due to their regular physical activity.
\end{abstract}

Key words: Body composition, dietary pattern, child nutrition, physical activity, obesity.

Este trabajo fue recibido el 13 de Octubre de 2009 y aceptado para ser publicado el 15 de Marzo de 2010.

\section{INTRODUCCIÓN}

En las últimas dos décadas se ha producido un cambio en el perfil epidemiológico- nutricional en Chile caracterizado por una disminución en la prevalencia de malnutrición por déficit y el aumento progresivo de la prevalencia de sobrepeso y obesidad. Prueba de esto es que el porcentaje de niños que asisten a primer año básico que son obesos aumentó de 5,5\% a 19,4\% entre los años 1987 y 2006, respectivamente $(1,2)$.

La obesidad se define como un desbalance entre la ingesta y el gasto energético, siendo mayor la energía consumida que la gastada por el organismo. En su etiología actúan factores genéticos y ambientales, jugando un rol esencial la alimentación y la actividad física (3-5).

La información respecto a los hábitos alimenta- rios e ingesta alimentaria en este grupo etáreo ha sido ampliamente estudiado en el extranjero (6-8) pero son escasos los estudios realizados en nuestro país, no existiendo datos de ningún tipo en niños que realicen alguna actividad física de manera sistemática. Los datos con los que contamos actualmente indican un alto consumo de meriendas y bebidas azucaradas junto con un bajo consumo de lácteos, frutas, verduras y legumbres $(5,9)$.

Datos respecto a la actividad física realizada por escolares muestran que existe una tendencia hacia actividades de tipo sedentarias, como videojuegos, computación y ver televisión. En Chile, recientemente se han incorporado dentro de los estudios la evaluación de la actividad física, encontrándose que niños entre 8 y 13 años de edad, pasan más de 3 horas realizando 
actividades de tipo sedentaria (ver televisión) las que aumentan 1 hora más en promedio los días de fin de semana, siendo este tiempo mayor en el grupo de niños con sobrepeso y obesidad (9). Estos resultados son similares a aquellos efectuados en el extranjero (6). Investigaciones más específicas para la evaluación de la capacidad física en niños chilenos han sido realizados por Kain y col. quienes mediante la aplicación de prueba físicos en escolares de escuelas públicas, obtuvieron como resultado una capacidad aeróbica disminuida en aquellos niños con obesidad comparados con los niños de peso normal (10). Adicionalmente, ha sido demostrado que niños obesos presentan un gasto energético en reposo menor que sus pares con peso adecuado (11).

La composición corporal en niños en edad escolar ha sido poco estudiada en Chile, por esto, la información disponible está basada en indicadores globales del estado nutricional sin indagar mayormente en los distintos componentes corporales, a pesar de la relación demostrada entre exceso de grasa corporal y desarrollo de enfermedades como aquellas enmarcadas en el síndrome metabólico (12-14).

El objetivo de este trabajo fue conocer la dieta consumida por escolares chilenos que realizaban una actividad física moderada en forma sistemática y determinar los diferentes componentes corporales en los grupos etáreos estudiados.

\section{SUJETOS Y MÉTODO}

Se evaluaron 105 escolares entre 11 y 14 años pertenecientes a la rama de fútbol del club deportivo Palestino S.A. y club deportivo Universidad Católica quienes en promedio realizan actividad física controlada y programada 8 horas por semana.

La evaluación antropométrica incluyó:

Peso: Se midió empleando una balanza SECA modelo 714 con precisión de $100 \mathrm{~g}$ (rango 0,1-130 kg.) ubicada en una superficie, horizontal y firme. La medición se realizó con el mínimo de ropa, con el sujeto ubicado en el centro de la plataforma con el peso distribuido uniformemente en ambos pies.

Talla: Se midió mediante tallímetro incorporado a la balanza SECA modelo 714 (rango $60-200 \mathrm{~cm}$.). El sujeto se colocó de pie, descalzo, con la cabeza en el plano de Frankfurt, que une el borde interior de la órbita de los ojos y el superior del meato auditivo externo, brazos a ambos lados del tronco, extendidos y con palmas tocando cara externa de los muslos, talones juntos tocando el extremo inferior de la superficie vertical con el borde interno de los pies en ángulo de 45 a 60 grados, zona occipital, escapular, nalgas, cara posterior de las rodillas y pantorrillas tocando superficie vertical del antropómetro.
Estado nutricional: Se determinó mediante el cálculo del IMC (peso/talla ${ }^{2}$ ) estimado por edad, comparándolo con las tablas de CDC/NCHS 2000. Los puntos de corte para clasificar el estado nutricional de los niños fue el recomendado por el comité de expertos en obesidad infantil de Norte América: bajo peso, IMC $\mathrm{p}<10$; normal, IMC $\mathrm{p} \geq 10-\mathrm{p}<85$; sobrepeso IMC $\mathrm{p} \geq$ 85- $\mathrm{p}<95$; obeso IMC $\mathrm{p} \geq 95$ (15).

Pliegues cutáneos: Las mediciones se realizaron en el lado derecho del cuerpo empleando un caliper Harpenden (rango 0-100 mm.), que ejerce una presión constante de 10 $\mathrm{g} / \mathrm{mm}^{2}$ de superficie de contacto. Para la determinación de la masa grasa se utilizó la fórmula de Slaughter que incluye tres variables: edad, pliegue tricipital y subescapular. Esta fórmula fue empleada ya que es la que presenta una mejor correlación con el gold estándar (DEXA) en este grupo etáreo comparado con otros métodos empleados (16). El pliegue tricipital se determinó en el punto medio entre acromion y olécranon, en la parte posterior del brazo, con el brazo relajado. El pliegue subescapular se midió en el punto localizado por debajo y por dentro de la escápula, con el hombro y el brazo relajado.

Encuesta alimentaria: Se aplicó una encuesta de registro alimentario de 24 horas, la que fue llenada por los niños en compañía de sus padres. Cada encuesta incluía un instructivo para el correcto registro de los alimentos, el cual fue revisado por el nutricionista encargado del proyecto. La encuesta fue cuantificada (17) y posteriormente analizada utilizando el software Food Processor 7.9 (ESHA Research, Oregon, USA) el que ha sido previamente validado con la incorporación de alimentos de consumo habitual entre la población Chilena (18).

El requerimiento energético fue calculado a partir de las recomendaciones para niños entre 1-18 años, FAO/ OMS/UNU de 2004, según la edad y nivel de actividad física (19) y posteriormente se comparó con la ingesta reportada con objeto de determinar adecuación energética. La ingesta de macro y micronutrientes se comparó con las recomendaciones internacionales para este grupo etario (20-23) calculando el porcentaje de adecuación para cada uno de estos.

Actividad física: Para conocer el nivel de actividad física se promedió la cantidad de horas de entrenamiento consideradas el plan de entrenamiento para cada serie, el que equivale en promedio a 6 horas de entrenamiento semanal más la competencia el fin de semana. En esta clasificación de nivel no se contempla actividad física realizada en el colegio, ni actividades recreativas.

Nivel socioeconómico: La clasificación socioeconómica se estableció por parte de los asistentes sociales de ambos clubes deportivos, usando el método de calificación socioeconómica ESOMAR (24). Este método, originario 
de Europa, se restringe a las variables posición laboral y educación, y ha sido validado previamente en Chile (25).

Análisis estadístico: El análisis estadístico de los datos se realizó empleando el software SPSS v 14.0 (Lead Technologies, USA). Una vez comprobado el comportamiento normal de los datos mediante el prueba Kolmogorov-Smirnov se aplicaron los prueba de comparaciones múltiples para poder determinar las diferencias entre los grupos evaluados, en aquellos datos normales se aplico análisis de la varianza (ANOVA) y posteriormente para determinar que grupo era causante de dicha diferencia se realizó el prueba de Bonferroni. Para el análisis de aquellos datos cuya distribución no fue normal se aplicó el prueba de Kruskall-Wallis.

\section{RESULTADOS}

Las características generales de la población estudiada se muestran en la tabla 1. El grupo de 11 años representó el 25,5\% de la muestra mientras que los niños de $12,13,14$ años representaron el 37,7\%; 17,9\% y el $18,9 \%$, respectivamente del total de la muestra estudia- da. Al comparar los promedios de peso, talla, IMC y masa grasa entre los cuatro grupos mediante ANOVA, encontramos que los valores promedios para cada una de estas variables fueron significativamente diferentes con un valor $\mathrm{p}<0,05$. Al corregir mediante el prueba de Bonferroni encontramos que el peso promedio en los cuatro grupos resultó ser significativamente diferente, mientras que el promedio de talla fue distinto en todos los grupos excepto entre los grupos de 12 y 13 años, el valor promedio IMC para niños de 11 años resultó estadísticamente diferente del grupo de 13 y 14 años, mientras que el IMC del grupo de 12 años resultó ser estadísticamente diferente del grupo de 14 años. Al comparar el \% de masa grasa encontramos que el valor promedio para el grupo de 14 años resultó ser significativamente menor del grupo de 11,12 y 13 años.

El 86\% de los niños presentaban un peso normal mientras que el $14 \%$ tenía sobrepeso, no encontrándose ningún caso de sobrepeso o bajo peso.

La tabla 2 muestra la ingesta promedio de energía y macronutrientes. Al comparar la ingesta energética

\section{TABLA 1}

\section{Características generales de la población en estudio (promedio \pm DE).}

\begin{tabular}{lccccc}
\hline Grupo & $\mathbf{n}$ & Peso $(\mathbf{k})$ & Talla $(\mathbf{m})$ & IMC & Masa grasa $(\%)$ \\
\hline 11 años & 27 & $37,3 \pm 5,1$ & $1,43 \pm 0,06$ & $18,2 \pm 1,7 * *$ & $14,5 \pm 3,3 * * *$ \\
12 años & 39 & $44,1 \pm 5,9$ & $1,53 \pm 0,08 *$ & $18,9 \pm 1,5 * *$ & $14,6 \pm 3,9 * * *$ \\
13 años & 19 & $49,1 \pm 6,4$ & $1,56 \pm 0,07 *$ & $20,0 \pm 1,8 * *$ & $15,5 \pm 3,0 * * *$ \\
14 años & 20 & $58,1 \pm 6,2$ & $1,66 \pm 0,07$ & $21,1 \pm 1,3$ & $11,7 \pm 4,5$ \\
$*$ No se encontró diferencia entre los grupos de 12 y 13 años de edad, p>0,05 \\
**No se encontró diferencias entre los grupos de 11 y 12 años, ni tampoco entre los grupos de 12 y 13 años de edad. p>0,05 \\
*** Los grupos de 11, 12 y 13 años resultaron ser estadísticamente distintos del grupo de 14 años de edad. p<0,05.
\end{tabular}

TABLA 2

Ingesta de macronutrientes en niños de 11 a 14 años

\begin{tabular}{|c|c|c|c|c|}
\hline & 11 Años & 12 Años & \multicolumn{2}{|c|}{ Aporte dietario } \\
\hline Calorías (kcal) & $2135 \pm 531 *$ & $2311 \pm 688$ & $2599 \pm 735$ & $2679 \pm 662 *$ \\
\hline Proteínas (g) & $82,8 \pm 19$ & $82,7 \pm 26,4$ & $89,7 \pm 28,5$ & $97,8 \pm 32,4$ \\
\hline H. de C (g) & $315,4 \pm 87,9$ & $352 \pm 112,2$ & $391,5 \pm 112,1$ & $407,8 \pm 105,7 *$ \\
\hline Fibra $(g)$ & $22,9 \pm 13,1$ & $21,2 \pm 12$ & $27,1 \pm 9,1$ & $23,2 \pm 9,6$ \\
\hline Grasa T (g) & $62,8 \pm 20$ & $64,8 \pm 25,7$ & $81,1 \pm 26,2$ & $75 \pm 27,9$ \\
\hline
\end{tabular}


y de macronutrientes entre los cuatro grupos etáreos se observa que el consumo aumenta progresivamente con la edad, sin embargo dicha diferencia resultó ser significativa solo entre las edades de 11 y 14 años para el consumo de calorías y carbohidratos, mientras que para la ingesta de proteínas y grasas totales no se encontraron diferencias significativas entre los grupos.

El aporte energético de macronutrientes fue similar en todos los grupos mostrando un aporte de carbohidratos, proteínas y grasas cercano al $60 \%, 15 \%$ y $26 \%$ respectivamente (datos no mostrados).

La ingesta de grasas y colesterol se muestra en la tabla 3. A pesar de que el consumo de grasas saturadas, monoinsaturadas, poliinsaturadas y colesterol aumenta con la edad, las diferencias observadas no son estadísticamente significativas. La relación entre los ácidos

\section{TABLA 3}

\section{Ingesta de grasas y colesterol en niños de 11 a 14 años}

\begin{tabular}{lcccc} 
& 11 Años & 12 Años & $\begin{array}{c}\text { 13 Años } \\
\text { Aporte Dietario }\end{array}$ & 14 Años \\
\hline Grasa Saturada (g) & $20,2 \pm 8,3^{*}$ & $22,1 \pm 9,8$ & $26,9 \pm 10,3$ & $24,7 \pm 11,8$ \\
Grasa monoinsaturada (g) & $21,6 \pm 8,1$ & $23,0 \pm 9,8$ & $28,7 \pm 7,7$ & $24,3 \pm 11$ \\
Grasa poliinsaturada (g) & $15,4 \pm 5,3$ & $15,2 \pm 6,9$ & $16,6 \pm 6,1$ & $18,6 \pm 5,4$ \\
Colesterol (mg) & $230,3 \pm 159,9$ & $253,2 \pm 215,7$ & $211,2 \pm 105,7$ & $259,1 \pm 180,4$ \\
Omega 3 (g) & $0,75 \pm 0,38$ & $0,72 \pm 0,38$ & $0,91 \pm 0,48$ & $0,87 \pm 0,56$ \\
Omega 6 (g) & $14,49 \pm 5,18$ & $14,29 \pm 6,77$ & $15,47 \pm 6,04$ & $17,48 \pm 5,2$ \\
Razón $\omega$-6: $\omega$-3 & 19,32 & 19,84 & 17,00 & 20,09 \\
\hline *(promedio \pm DE). & \multicolumn{4}{c}{}
\end{tabular}

FIGURA 1

Adecuación de calorías y macronutrientes en niños de 11 a 14*

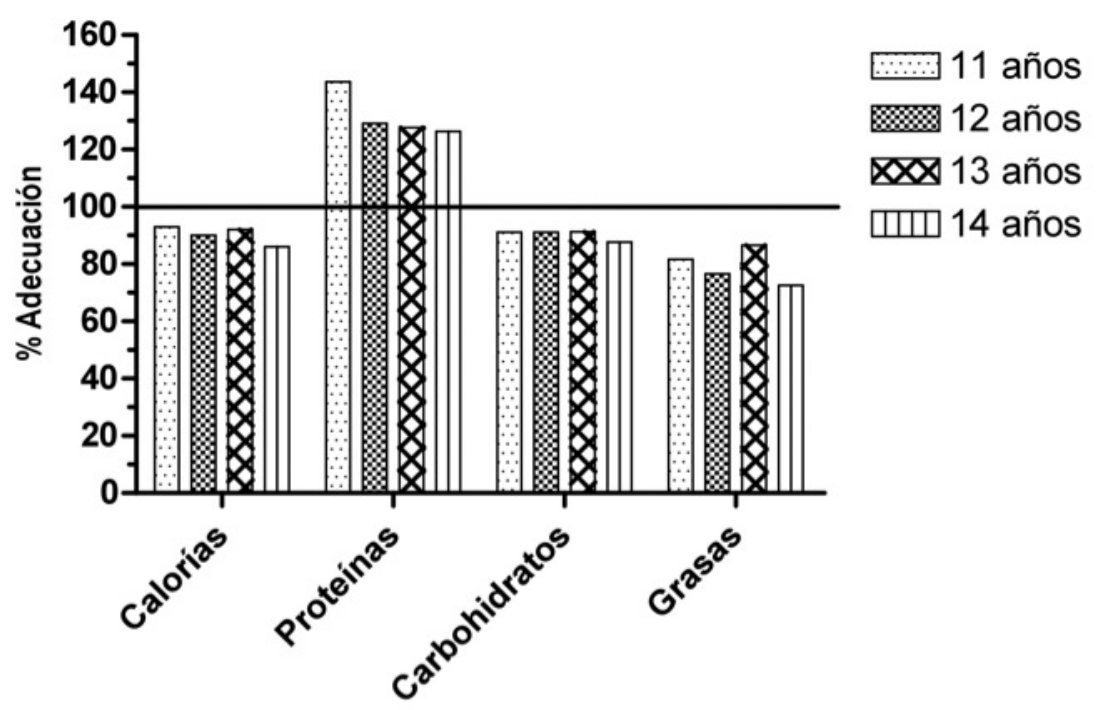

* Adecuación respecto de las recomendaciones (19).

No se encontraron diferencias significativas entre las distintas edades en cada grupo de macronutrientes. 
grasos omega 3/omega 6 fue de 19:1 en los cuatro grupos evaluados, mucho mayor que la recomendación actual de 10:1 para la población chilena (26).

La figura 1 muestra el grado de adecuación de la dieta en calorías ajustadas por actividad física y macronutrientes. En promedio, la ingesta de calorías, carbohidratos y grasas fue menor que el recomendado para la edad en los cuatro grupos, siendo cercano al $90 \%$ de la recomendación en calorías e hidratos de carbono, y de aproximadamente un $80 \%$ para grasas totales. La menor adecuación de energía y macronutrientes se observó en el grupo de 14 años.

Al analizar el consumo de micronutrientes en los cuatro grupos estudiados encontramos que el consumo de niacina en los niños de 14 años resultó ser significativamente mayor que el consumo de niacina de los niños

\section{FIGURA 2}

Adecuación de micronutrientes seleccionados en niños de 11 a 14 años*
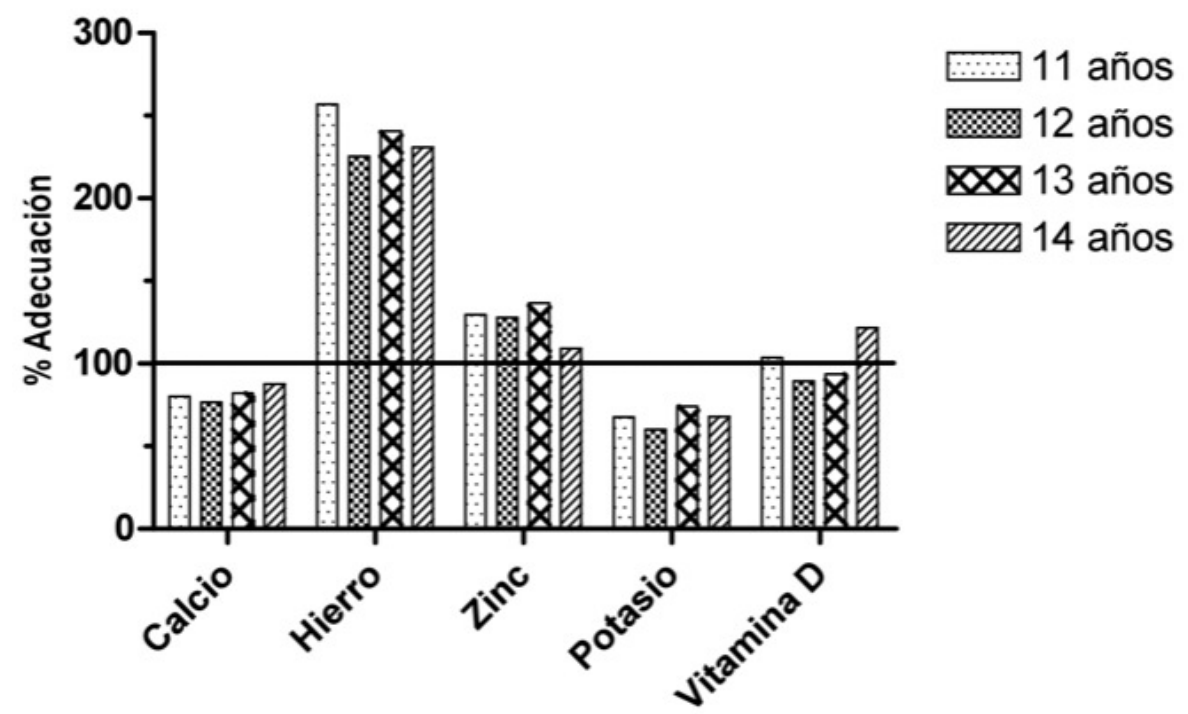

* Adecuación respecto de las recomendaciones (19).

No se encontraron diferencias significativas entre las distintas edades en cada grupo de macronutrientes.

\section{TABLA 4}

Consumo de alimentos en niños de 11 a 14 años.

\section{Grupo alimentario (gramos/día)}

11 años

12 años

13 años

14 años

Cereales

Frutas

Verduras

Lácteos

Carnes

Aceites

Azúcares

$$
312,5 \pm 128,1^{*}
$$

$181,79 \pm 156,8$

$170,57 \pm 136,3$

$514,46 \pm 253,9$

$145,39 \pm 66,9$

$43,46 \pm 34,2$

$49,11 \pm 45,3$

$$
\begin{gathered}
398,45 \pm 141,7 \\
184,23 \pm 162,1 \\
102,1 \pm 76,9 \\
489,92 \pm 320,5 \\
139,10 \pm 80,2 \\
37,04 \pm 31,7
\end{gathered}
$$$$
56,6 \pm 54,4
$$

$$
\begin{gathered}
479,74 \pm 166,2 * \\
186,84 \pm 206,3 \\
139,47 \pm 130,7 \\
525,53 \pm 381,7 \\
146,58 \pm 76,5 \\
57,53 \pm 25,8
\end{gathered}
$$$$
66,4 \pm 40,8
$$

$443,3 \pm 95,2 *$

$210,0 \pm 265,9$

$162,0 \pm 161,8$

$618,75 \pm 407,2$

$148,75 \pm 84,9$

$52,20 \pm 61,1$

$43,95 \pm 27,8$

ANOVA $* \mathrm{p}<0,05$ (Promedio $\pm \mathrm{DE}$ ) 
entre 11 y 12 años $(\mathrm{p}<0,009)$ y el consumo de vitamina $E$ en el grupo de 14 años fue mayor que el de los niños de 12 años $(p<0,008)$. Para el resto de los micronutrientes analizados no se encontraron diferencias significativas (datos no mostrados).

Para evaluar la adecuación de micronutrientes se seleccionaron aquellos nutrientes característicamente deficitarios en poblaciones de edades similares. La figura 2 muestra que la ingesta promedio de calcio y potasio no alcanzó la recomendación en ninguna de las edades, mientras que la ingesta de hierro, zinc y vitamina $\mathrm{D}$ fue suficiente.

Observamos en la tabla 4 el consumo diario promedio de alimentos. Si bien existen diferencias en el consumo entre las diferentes edades, solo resulta ser significativo el menor consumo de cereales entre niños de 11 años con respecto a los de 13 y 14 años. En general se observa en la muestra un bajo consumo de frutas, verduras y lácteos cuyos valores promedio representan un consumo diario de $1 \frac{1 / 2}{2}$ fruta, $1 \frac{1 / 2}{2}$ a 2 platos de verduras y $21 / 2$ a 3 vasos de leche, mientras que el consumo de cereales (representado principalmente por pan blanco) alcanza de 3 a 4 unidades diarias (300-400 g)

Al comparar las ingesta promedio de los diferentes grupos de alimentos con respecto a la recomendación alimentaria para la población chilena (figura 3), se ob- serva que el consumo de cereales fue adecuado en todos los grupos estudiados, siendo en las edades extremas cercano al 95\% mientras que entre los niños de 12 y 13 años el consumo sobrepasa la recomendación (26). En todos los grupos se observa una ingesta deficiente de frutas, verduras y lácteos, para éste último mostró una ingesta adecuada sólo el grupo de 14 años. Por otra parte, en los cuatro grupos etáreos evaluados, solo un $35 \%$ y $30 \%$ de los niños alcanzó la recomendación de consumo diaria de frutas y verduras, mientras que las carnes y aceites presentaron una ingesta adecuada en todos los grupos estudiados.

\section{DISCUSIÓN}

En nuestro país no contamos con encuestas alimentarias recientes de alimentación que abarquen grandes grupos de población, contándose sólo con datos de investigaciones en grupos pequeños que nacen del interés de grupos particulares de investigación, además no existen encuestas en niños que realicen actividad física de 8 horas por semana.

Dado que la muestra en este estudió estuvo formada por niños en edad escolar que realizaban ejercicio físico en promedio 8 horas a la semana, se encontraron bajas tasas de sobrepeso y nula de obesidad, contrastando con antecedentes publicados de escolares sedentarios

\section{FIGURA 3}

\section{Adecuación consumo de alimentos en niños de 11 a 14 años*}

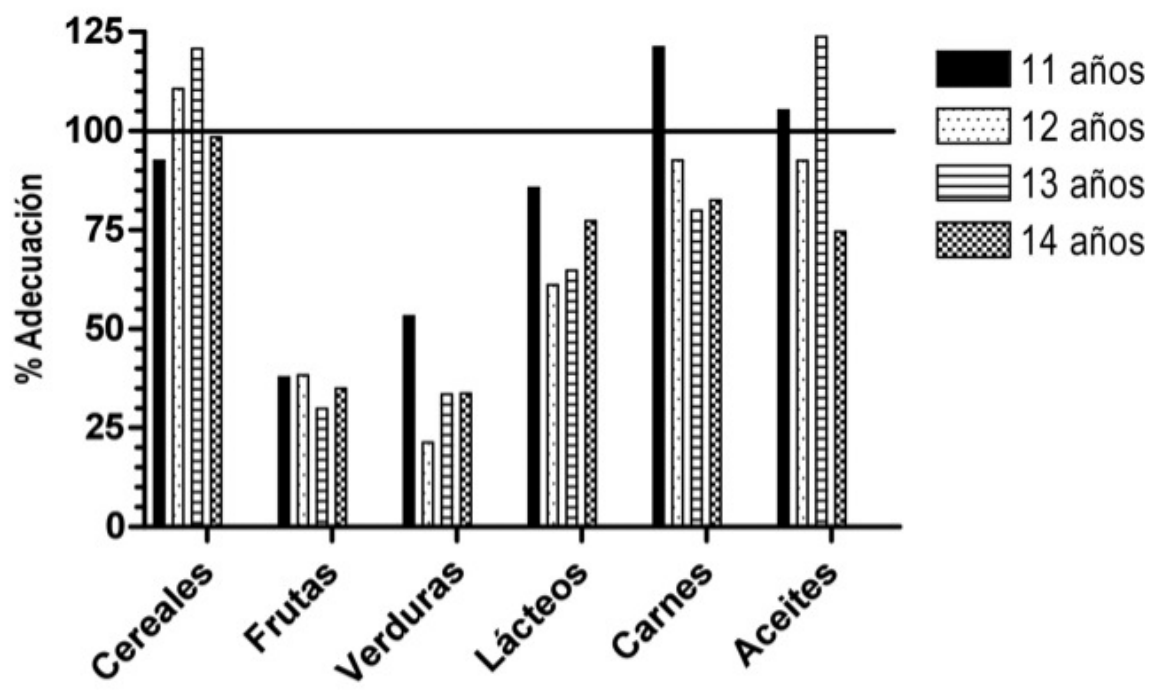

* Adecuación respecto de las recomendaciones (19).

No se encontraron diferencias significativas entre las distintas edades en cada grupo de macronutrientes. 
$(27,28)$.

La última encuesta nacional de salud realizada en Estados Unidos (NHANES III 1999-2002) (27) mostró una prevalencia de sobrepeso de $19,9 \%$ y $18,3 \%$ en niños de 6-11 años y 12-19 años respectivamente, similares a los encontrados en estudios realizados en Europa. Zimermann y colaboradores evaluaron a 2.431 niños suizos entre 6 a 12 años (50\% hombres) en el 2004 encontrando una prevalencia de sobrepeso y obesidad de un $19,9 \%$ y $7,4 \%$ respectivamente (29). Otro estudio realizado por Karayiannis y colaboradores en una muestra mayor de niños griegos entre 11 y 15 años de edad ( $n=4299$ niños; $48,7 \%$ hombres) halló una prevalencia de $18,8 \%$ de sobrepeso y $5,8 \%$ de obesidad (30).

En Latinoamérica la situación no difiere de la realidad mundial, en Brasil la prevalencia de sobrepeso en niños de 6-18 años alcanza al 13,1\%, mientras que datos arrojados por la encuesta nacional de nutrición en México, donde se estudio un total de 9.259 niños 5 a 11 años, fue de un 19,5\% para sobrepeso y obesidad en conjunto. En la provincia de Corrientes, Argentina, Poletti y Barrios evaluaron a 3.931 niños de 6 a 14 años de los cuales 55,3\% eran hombres, encontrando un $13,6 \%$ de sobrepeso y $8,4 \%$ de obesidad, por otra parte Kovalsky y colaboradores estudiaron 1.289 niños argentinos de 10-19 años (37,4\% hombres) de los cuales un $24,1 \%$ presento sobrepeso y $7,9 \%$ obesidad (31-34).

Estudios realizados en escolares de nuestro país ( $n=1701,54,4 \%$ hombres) encontraron una prevalencia de sobrepeso de $18,6 \%$ y de un $15,4 \%$ de obesidad en edades de 10 a 13 años (9).

Investigaciones en grupos etáreos menores (estudiantes cuarto año básico) han encontrado una prevalencia semejante a la del grupo anteriormente señalado, alcanzando un $17,3 \%$ para sobrepeso y $17,7 \%$ en obesidad (35). Otro estudio, realizado en la región metropolitana, en niños de nivel socioeconómico alto (2 a 18 años de edad) encontró una prevalencia de 14\% y $13 \%$ de sobrepeso y obesidad respectivamente (36).

En relación a la evaluación de composición corporal, no se puede establecer una comparación con datos previamente evaluados, dado que los métodos utilizados en diferentes estudios varían considerablemente. Estudios realizados en Estados unidos, evaluando niños blancos, negros e hispánicos entre 3 y 18 años mediante DEXA, mostró que los niños entre 10 y 14 años de origen hispánicos, tenían un $26.0 \%$ de masa grasa (37), cantidad considerablemente mayor a la evaluada en nuestra muestra.

La ingesta energética y de los macronutrientes cubrió los requerimientos de los grupos estudiados, encontrándose una adecuación superior al $100 \%$ en proteínas, cercano al $90 \%$ en energía y carbohidratos y de alrededor de $80 \%$ en grasas, con una ingesta de colesterol inferior a $300 \mathrm{mg} / \mathrm{día}$. La razón de ingesta de ácidos grasos $\omega$-6: $\omega$-3 fue de 19,1, muy lejana a las recomendaciones establecidas (10:1) dato similar al encontrado en otro estudio en el extranjero (38).

El patrón de alimentación encontrado es este estudio se debe a las características propias de esta cohorte, ya que por tratarse de futbolistas que reciben educación alimentaria en sus clubes, además de estar imbuidos en un ambiente deportivo, reciben información sobre la alimentación adecuada para la realización de actividad física, recalcándose habitualmente en el ambiente deportivo el consumo de proteínas y la disminución del consumo de grasas. Por esto resulta interesante que la distribución porcentual de la energía total aportada por los diferentes macronutrientes no difiere mayormente de otras encontradas en grupos de niños no deportistas (39).

La ingesta de micronutrientes, en general baja, es insuficiente para cubrir las recomendaciones de ingesta de calcio y potasio micronutrientes importantes en sujetos que realizan actividad física, debido a la importancia de la densidad mineral ósea y balance hidroelectrolítico, esto se relaciona con la menor ingesta de lácteos, frutas y verduras del grupo en estudio, lo que se aprecia en la mayoría de los estudios realizados en escolares tanto en nuestro país como en el extranjero (40-41).La ingesta de frutas, verduras y lácteos, aunque mayor que la reportado por otros autores en poblaciones similares (35), es insuficiente respecto de las recomendaciones del Ministerio de Salud para estos grupos etéreos. Un estudio realizado en escolares chilenos de 8 a 13 años reportó un consumo promedio de 2,4 a 2,8 porciones/día para fruta y verduras y de 1,2 a 1,5 porciones/día para lácteos, también insuficientes respecto de las recomendaciones (9). Resultados similares han sido hallados por Brady y colaboradores en niños norteamericanos, donde el promedio de porciones/días de frutas, verduras y lácteos, consumidas por niños entre 7 y 14 años era de 0,$9 ; 2,4$ y 1,2 porciones/día respectivamente, lo que esta por debajo de las recomendaciones indicadas por las guías de alimentación para la población desarrolladas por el Departamento de Agricultura de los Estados Unidos (41).

El consumo de cereales reportado aquí fue de un $55,6 \%$ mayor a lo recomendado, siendo el consumo de pan fue responsable de un $70 \%$ de la ingesta de cereales, hecho que coincide con datos publicados en la literatura por Yáñez y colaboradores quienes evaluaron el consumo de alimentos en una muestra de 249 niños de $5^{\circ}$ a $8^{\circ}$ año básico de ambos sexos en tres comunas de nuestro país, mostrando unas ingesta un $57,4 \%$ mayor que las recomendaciones (42).

El consumo de carne en el 75\% de la población eva- 
luada fue adecuado con respecto a lo recomendado en las guías de alimentación para la población chilena, mientras que para grasa fue del $57 \%$, los valores obtenidos para el consumo de cárneos concuerdan con lo reportados por Atalah en escolares de Aysén, sin embargo, en estos mismos escolares el consumo adecuado de aceites y grasas alcanzó solo al 27\%, situación que fue corroborada por Yáñez y colaboradores $(5,42)$.

El consumo de azúcar entre los grupos evaluados expresado como aporte calórico/ día va desde 175,8 a $265,6 \mathrm{Kcal} . /$ día, lo que representa menos del $10 \%$ de las calorías totales. Estos valores difieren considerablemente de los publicados por Yánez y colaboradores en donde un 63,9\% de los escolares presentó un consumo excesivo y de los reportados por Kain y Andrade en preescolares con un consumo de 350-500 kcal. (42,43). Como se mencionó anteriormente, esta ingesta más baja se debe al mejor nivel de educación nutricional de los niños es nuestro estudio.

El 37\% de la población consumió algún tipo de meriendas, siendo el de mayor frecuencia de consumo las galletas, queques y chocolates.

\section{CONCLUSIONES}

A la luz de los resultados obtenidos en el presente estudio podemos concluir que la práctica regular y sistemática de actividad física resulta ser de gran importancia en la prevención del sobrepeso u obesidad, incluso cuando los patrones alimentarios son similares a los descritos para niños sedentarios chilenos de la misma edad que muestran una alta prevalencia relativa de sobrepeso y obesidad.

Dado que el grupo estudiado es constantemente aconsejado respecto a la alimentación en la práctica deportiva es que encontramos ingestas menores de ciertos grupos de alimentos, que tradicionalmente su consumo es elevado en este grupo etáreo, tal es el caso de azúcares y grasas.

También es importante destacar que si bien las adecuaciones en algunos grupos de alimentos resultaron ser insuficientes, debido a un mayor requerimiento energético del grupo estudiado, estas son mayores que las descritas en la literatura para niños de la misma edad.

Este estudio muestra que la práctica regular de actividad física prácticamente elimina la obesidad y disminuye marcadamente el sobrepeso, hecho importante a considerar en la educación básica en nuestro país.

\section{RESUMEN}

Existe escasa información en nuestro país sobre la alimentación de diferentes grupos de la población. El objetivo del estudio es conocer la dieta consumida por escolares que efectúan actividad física regularmente y determinar su composición corporal. Se evaluaron 105 escolares entre 11 y 14 años pertenecientes a dos clubes deportivos, quienes en promedio realizan actividad física programada 8 horas por semana. La evaluación antropométrica incluyó peso, estatura, pliegue tricipital y subescapular, además de un registro alimentario de 24. Se evaluó el nivel socioeconómico mediante la encuesta ESOMAR. Como era de esperar el peso y la talla aumentaron progresivamente con la edad. El porcentaje de grasa corporal aumentó entre 11 y 13 años de edad para luego descender a los 14 años. Un $86 \%$ presentó un estado nutricional normal y $14 \%$ sobrepeso. No se observó obesidad en este grupo de niños. Tanto la ingesta energética como los macronutrientes fueron suficientes en todos los grupos, encontrándose déficit solo para calcio y potasio que se asocia a baja ingesta de lácteos, frutas y verduras. En conclusión, este grupo presenta patrones similares de consumo alimentario que escolares de la misma edad, sin embargo presentan mejor estado nutricional, probablemente debido al mayor nivel actividad física.

Palabras clave: composición corporal; dieta; alimentación niños, actividad física, obesidad.

Dirigir la correspondencia a:

Dr.

Jaime Rozowski Narkunska

Departamento de Nutrición, Diabetes

y Metabolismo.

Escuela de Medicina

Pontificia Universidad Católica de Chile

Lira 40

Santiago

Teléfono: 3543857

E-mail: irozowski@puc.cl

\section{BIBLIOGRAFÍA}

1. Situación de_hesidad _ $1^{\circ}$ hásico por región Disponible en h tp / / www sistemas junaeb cl/estados. nutricionales 2007/tablaobesidadregion. Acceso Diciembre 06, 2007.

2. Vio F, Albala C: Nutrition policy in the Chilean transition. Public Health Nutr 2000;1: 49-55.

3. Barlow S, Dietz W: Obesity evaluation and treatment: expert committee recommendation. Pediatrics 1998; 102: 1-11.

4. Burrows R, Díaz N, Muzzo S. Variaciones del índice de masa corporal (IMC) de acuerdo al grado de desarrollo puberal alcanzado. Rev Med Chil 2004; 132 (11): 1363-1368.

5. Atalah E, Urteaga C, Rebolledo A et al. Patrones 
alimentarios y de actividad física en escolares de la Región de Aysén. Rev Chil Pediatr 1999; 70 (6): 483-490.

6. Dehghan M, Akhtar-Danesh N, Merchant A: Childhood obesity, prevalence and prevention Nutr J 2005, 4:24.

7. Crespo C, Smit E, Troiano R, Bartlett S, Macera C, Anderson R: Television watching, energy intake and obesity in US children. Arch Pediatr Adolesc Med 2001; 155: 360-365

8. Fernández J: Dietary habits and nutritional status of school aged children in Spain. Nutr. Hosp 2006; 21 (3): 374-378.

9. Olivares S, Kain J, Lera L, F Pizarro F, Vio F, Moro C, Nutritional status, food consumption and physical activity among Chilean school children: a descriptive study. European J Clin Nutr 2004; 58: 1278-1285.

10. Kain J, Olivares S, Romo M, Leyton B, Vio B, Cerda R, González R, Giadalah A, Albala C. Estado nutricional y resistencia aeróbica en escolares de educación básica: línea base de un Proyecto de Promoción de la Salud. Rev Méd Chile 2004; 132: 1395-1402.

11. Carrasco F, Reyes E, Charlin V, Kehr J: Gasto energético de reposo medido versus gasto energético por fórmula en niños obesos. Rev Chil Pediatr 1999; 70: 107-112.

12. Maynard M, Wisemandle W, Roche AF, Chumlea C, Guo S, Siervogel R: Childhood Body Composition in Relation to Body Mass Index: Pediatrics 2001; $107 ; 344-350$

13. Janicka A, Tishya A. L. Wren, Sanchez M, Dorey F, Kim PS, Mittelman SD, Gilsanz V: Fat Mass Is Not Beneficial to Bone in Adolescents and Young Adults. J Clin Endocrinol Metab 2007; 1: 143-147

14. Perichart-perera O, Balas-nakash M, Schiffmanselechnik E, Barbato-dosala, Vadillo-ortega F: Obesity Increases Metabolic Syndrome Risk Factors in School-Aged Children from an Urban School in Mexico City. J. Am. Diet. Assoc. 2007; 107: 81-91.

15. CDC/NCHS (2000): CDC growth charts: United States. http://www.cdc.gov/growthchart Posted May 30,2000 on the Internet

16.

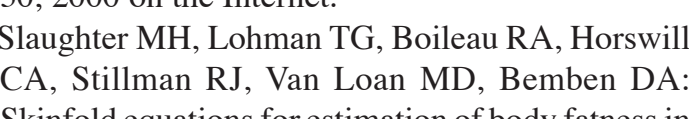
Skinfold equations forestimation of body fatness in children and youth. Hum Biol 1988; 60 (5): 709-23.

17. Jury G, Urteaga C, Taibo M. Porciones de intercambio y composición química de los alimentos de la pirámide alimentaria chilena. INTA, Centro de Nutrición Humana, Facultad de Medicina, Uni- versidad de Chile. 1997.

18. Castillo O, Rozowski J, Muñoz X, Cuevas A, Maiz A, Urquiaga I, Leighton F. Ingesta de nutrientes en vegetarianos chilenos. Rev Chil Nutr 1998; 25 : 39-44.

19. Human energy requirements, Report of a Joint FAO/ WHO/UNU Expert Consultation Rome, 17-24 October 2001.

20. Food and Nutrition Board. Dietary Reference Intakes for calcium, Phosphorus, Magnesium, Vitamin D and Fluoride. National Academy Press, Washington D.C. 1997.

21. Food and Nutrition Board. Dietary Reference Intakes: Thiamin, Riboflavin, Niacin, Vitamin B6, Folate, Vitamin B12, Pantothenic Acid, Biotin and Choline. National Academy Press, Washington D.C. 1998.

22. Food and Nutrition Board. Dietary Reference Intakes: Vitamin A, Vitamin K, Arsenic, Boron, Chromium, Copper, Iodine, Iron, Manganese, Molybdenum, Nickel, Silicon, Vanadium and Zinc. National Academy Press, Washington D.C 2001.

23. Food and Nutrition Board. Dietary Reference Intakes: Vitamin C, Vitamin E, Selenium and Carotenoids. National Academy Press, Washington D.C 2000.

24. European Society for Opinion and Marketing Research. Standard demographic classification. ESOMAR 1997.

25. Méndez R. El nivel socioeconómico Esomar. VIII Congreso Chileno de Marketing de Icare. Junio 1999.

26. Castillo C, Uauy R, Atalah E, Eds. Guías de alimentación para la población Chilena, La Nación, 1997.

27. Cynthia L. Ogden, Margaret D. Carroll, Lester R. Curtin, Margaret A. McDowell, Carolyn J. Tabak, Katherine M. Flegal. Prevalence of Overweight and Obesity in the United States, 1999-2004 JAMA 2006; 295: 1549-1555.

28. Liberona Y, Engler V, Castillo O, Rozowski J. "Ingesta de macronutrientes y prevalencia de malnutrición por exceso en escolares de $5^{\circ}$ y $6^{\circ}$ básico de distinto nivel socioeconómico de la Región Metropolitana”. Rev Chil Nutr 2008; 35: 190-199.

29. Zimmermann MB, Gübeli C, Püntener C, Molinari $\mathrm{L}$; Overweight and obesity in 6-12 year old children Switzerland. Swiss Med Wkly 2004; 134 (35-36): $511-3$

30. Sarayiannis D
sis LS, Kokkevi A. Prevalence of overweight and
obesity in Greek school-aged children and adoles-
cents. Eur J Clin Nutr 2003; 57 (9): 1189-92. 
31. Wang Y, Monteiro C, Popkin B: Trends of obesity and underweight in older children and adolescents in the United States, Brazil, China, and Russia. Am J Clin Nutr 2002; 75: 971-7.

32. Hernández B, Cuevas-Nasu L, Shamah-Levy T: Factores asociados con sobrepeso y obesidad en niños mexicanos de edad escolar: resultados de la Encuesta Nacional de Nutrición 1999. Salud Pública Méx 2003; 45: S551-S557.

33. Poletti OH, Barrios ML. Sobrepeso y obesidad como componentes de la malnutrición, en escolares de la ciudad de Corrientes, Argentina. Rev Chil Pediatr 2003; 74: 499-503.

34. Kovalskys, Irina, Bay, Luisa, Rausch Herscovici, Cecile et al. Prevalencia de obesidad en una población de 10 a 19 años en la consulta pediátrica. Rev Chil Pediatr 2005; 76 (3):.324-325.

35. Olivares S, Zacarías I, Lera L, Leyton B, Durán R, Vio F: Estado nutricional y consumo de alimentos seleccionados en escolares de la región metropolitana: Línea base para un proyecto de promoción del consumo de pescado. Rev Chil Nutr 2005: 32 (2): 102-108.

36. Eyzaguirre F, Mericq V, Ceresa S, Youlton R, Zacarías J: Prevalencia de sobrepeso y obesidad en niños que se controlan en pediatría ambulatoria en Clínica Las Condes: Rev Chil Pediatr 2005; 76
(2): 143-149.

37. Ellis K. Body composition of a young, multiethnic, male population. Am J Clin Nutr 1997: 66: $1323-31$.

38. Oddy W, de Klerk N, Kendall G, Mihrshahi S, Peat J. Ratio of omega- 6 to omega-3fatty acids and childhood asthma J Asthma 2005:41(3) 319 - 326

39. Hanning RM, Waodruff SJ, Lambraki I, Jessup L, Driezen P, Murphy CC: Nutrient intakes and food consumption patterns among Ontario students in grades six, seven, and eight. Can J Public Health 2007; 98 (1): 12-6.

40. Muñoz, K; Krebs-Smith S, Ballard-Barbash R, Cleveland L: Food Intakes of US Children and Adolescents Compared With Recommendations Pediatrics 1997; 100: 323-329.

41. Brady L, Lindquist C, Herd S, Goran M; Comparison of children's dietary intake patterns with US dietary guidelines. British J Nutr 2000; 84: 361-367

42. Yáñez R, Olivares S, Torres I, Guevara M, Díaz Nora: Consumo de alimentos en escolares chilenos, Su relación con las guías y la pirámide alimentaría. Rev Chil Nutr 2001; 28: 422-428.

43. Kain J, Andrade M: Charasteristics of the diet and patterns of physical activity in obese Chilean preschoolers. Nutr Res 1999; 18: 1825-1835. 